\title{
ORIGINAL
}

\section{TASA DE DEPENDENCIA DE LA POBLACIÓN ESPAÑOLA NO INSTITUCIONALIZADA Y CRITERIOS DE VALORACIÓN DE LA SEVERIDAD (*)}

\author{
Antonio Alegre Escolano (2), Mercedes Ayuso Gutiérrez (2), Montserrat Guillén Estany (2), \\ Malena Monteverde Verdenelli (1) y Enrique Pociello García (2) \\ (1) Departamento de Matemática Económica, Financiera y Actuarial, Universidad de Barcelona \\ (2) Departamento de Econometría, Estadística y Economía Española, Universidad de Barcelona \\ (*) Para la realización del mismo se ha contado con la ayuda FEDER a través de los proyectos SEC2001-3707 / 3672 \\ / 2581 y SEJ2004-05052, así como de la Fundación BBVA.
}

\section{RESUMEN}

Fundamento: El criterio de valoración del grado de severidad de la dependencia influye en la estimación de los costes de los servicios de cuidados de larga duración, especialmente en la población de 65 y más años. El objetivo de este trabajo es analizar las diferencias en la tasa de dependencia en personas con discapacidades de carácter permanente dependiendo del criterio de severidad utilizado.

Métodos: Se utilizan los datos de la Encuesta sobre Discapacidades, Deficiencias y Estado de Salud recogidos por el Instituto Nacional de Estadística en 1999. Se comparan dos criterios de valoración de la severidad de las discapacidades padecidas por un individuo. Se ajusta el logaritmo de las tasas de prevalencia por edad, para cada sexo y se contrasta estadísticamente la existencia de diferencias significativas entre los criterios a partir de intervalos de confianza al $95 \%$.

Resultados: El criterio basado en la máxima severidad (empleado por el Instituto Nacional de Estadística) infravalora entre 10\% $25 \%$ el grado de dependencia absoluto medido por el criterio alternativo, sobrevalorando hasta un $25 \%$ los grados de dependencia moderados a partir de los 65 años. En la población masculina ese mismo criterio produce una infravaloración del $10 \%$ en la dependencia grave a partir de los 95 años.

Conclusiones: El criterio de máxima severidad utilizado por el Instituto Nacional de Estadística infravalora la tasa de dependencia más grave, especialmente a partir de los 80 años.

Palabras clave: Actividades cotidianas. Dependencia. Personas con discapacidad. Anciano. Salud del anciano. Envejecimiento de la población.

Correspondencia:

Malena Monteverde Verdenelli

Departamento de Econometría, Estadística y Economía Española

Facultad de Ciencias Económicas y Empresariales

Universidad de Barcelona

Av. Diagonal 690

08034 Barcelona

Correo electrónico: mmonteverde@ub.edu, tel

\section{ABSTRACT \\ Dependency Rate among Spain's Non-Institutionalized Population and Criteria for Evaluating Severity}

Background: The criteria for evaluating the degree of severity of dependence has an impact on estimating the costs of the longterm care services, especially among the population over 65 years of age. The sensitivity of the dependency rate must be analyzed in view of different definitions of seriousness, whether by the maximum severity observed in the disability in activities of daily living or by the number and severity of all these disabilities.

Methods: The data from the Disability, Deficiency and Health Condition Survey conducted by the Spanish National Institute of Statistics in 1999 are used. A comparison is drawn between two criteria for evaluating the severity of an individual's disabilities. Models are made with the logarithms of the prevalence rates by age, for each sex, a comparison of the existence of significant differences between these criteria being drawn based on $95 \%$ confidence intervals.

Results: The criterion based on the maximum severity (employed by the Spanish National Institute of Statistics) underestimates the degree of total dependency measured by the alternative criterion by $10 \%-25 \%$, overestimating the degrees of moderate dependency as of age 65 by up to $25 \%$. Among the male population, this same criterion gives rise the medium dependency as of age 95 being underestimated by $10 \%$.

Conclusions: The maximum severity criterion employed by the Spanish National Institute of Statistics underestimates the most serious dependency rate, especially as of 80 years of age.

Key words: Activities of daily living. Disabled persons. Aging health. Aged. Demographic Aging. 


\section{INTRODUCCIÓN}

El fenómeno de la dependencia, presente en la actualidad en la mayor parte de las sociedades occidentales, es especialmente acentuado en España ${ }^{1-3}$, donde el envejecimiento cobra mayor importancia debido a la extraordinaria disminución de la fecundidad y al aumento de la esperanza de vida. Actualmente la población de 65 años y más asciende aproximadamente al $17 \%$ de la po blación española, lo que significa más de 6,8 millones de personas. Se estima que en el año 2050 dicho segmento de edad constituirá más del $30 \%$ de la población, con 16,4 millones de personas ${ }^{4}$.

Según la definición del Consejo de Euro$\mathrm{pa}^{5}$ de 1998, la dependencia es el estado en el que se encuentran las personas que, por razones ligadas a la falta o pérdida de autonomía física, psíquica o intelectual, tienen necesidad de asistencia y/o ayuda importantes para realizar las actividades de la vida cotidiana. Y según el mismo organismo: si bien la dependencia no es una situación exclusiva de las personas mayores, si es cierto que su incidencia aumenta con la edad. De forma similar, la oficina del Defensor del Pueblo ${ }^{6}$ definió en el año 2000 la dependencia como la necesidad de atención y cuidados que precisan las personas que no pueden hacer por sí mismas las actividades de la vida cotidiana. Para otras mediciones de discapacidad y un estudio sobre sus causas y factores de riesgo se pueden consultar los trabajos de Viciana, Hernández-Rodríguez, Conto y Ávila ${ }^{7}$ y Verbrugge y Jette ${ }^{8}$, respectivamente.

De acuerdo con la Encuesta de Discapacidades, Deficiencias y Estado de Salud, realizada en España en 1999, del total de personas de más de 65 años, alrededor de 1,5 millones, padecen discapacidades para realizar actividades de la vida diaria, lo que representa que casi una de cada cuatro personas mayores de 65 años tiene algún grado de dependencia. Para el futuro ${ }^{4}$ se prevé que este escenario demográfico no sólo se mantenga sino que se acentúe, con el consiguiente aumento del gasto en los servicios destinados al cuidado de este segmento de la población.

Los resultados arrojados por un trabajo publicado recientemente a partir de un estudio longitudinal realizado en Leganés ${ }^{9}$, indican que la aparición de la dependencia se produce en edades más avanzadas y que ello implica una mayor esperanza de vida libre de discapacidad, pero también una mayor complejidad de los cuidados necesarios para las poblaciones dependientes, que están constituidas fundamentalmente por mujeres muy ancianas ${ }^{10}$.

Ante el interrogante que suscita la sostenibilidad de los servicios públicos necesarios para cubrir las demandas de cuidados de larga duración, la cobertura mediante seguros se perfila como una alternativa, o medida complementaria, cada vez más clara en nuestro país. Es lo que viene denominándose «Seguro de Dependencia», desarrollado en algunos países como en Estados Unidos, bajo sistemas privados, y en otros, como en Alemania, como un seguro público ${ }^{11}$.

Para poder estimar con cierta precisión el impacto que el crecimiento de la población dependiente tiene y tendrá sobre la demanda de servicios de cuidados de larga duración es necesario avanzar en el análisis cuantitativo y cualitativo de este colectivo. Por un lado, la medición de la dependencia requiere de la definición precisa del término y, por otro, es fundamental un criterio de valoración del grado de severidad de la falta de autonomía.

Respecto a este criterio podemos preguntarnos: ¿Cuál es la diferencia entre utilizar como criterio de valoración del grado de dependencia la máxima severidad en las discapacidades padecidas por un individuo (criterio utilizado por el $\mathrm{INE}^{12}$ ) o algún otro criterio que además de la severidad contemple 
el número de discapacidades que padece el individuo? Nos referimos con esta segunda alternativa a criterios similares al utilizado por Pitacco ${ }^{13}$ para Italia en el ámbito asegurador y que están más cercanos a índices hasta el momento utilizados en gerontología, como el índice de Barthel $^{14}$ y el propuesto por Bone ${ }^{15}$ con el objeto de medir la tendencia de la dependencia en la población del Reino Unido.

El objetivo de este trabajo es analizar las diferencias de la tasa de dependencia en la población española de personas con discapacidades de carácter permanente dependiendo del criterio de severidad utilizado.

\section{MATERIAL Y MÉTODOS}

Dado que para este trabajo se van a analizar las discapacidades de carácter permanente, se consideraran las dificultades que son padecidas o se espera que sean padecidas por los individuos durante más de un año.

Con el fin de identificar los distintos niveles de dependencia del colectivo de personas mayores con discapacidades, interesa analizar la dependencia de las personas con discapacidades relacionada con los cuidados de larga duración, es decir la dependencia que tienen de otras personas y de ahí que no se incluya a las personas con discapacidad que han superado la dificultad para realizar la actividad por medio del uso de alguna ayuda técnica.

Además, en el presente estudio nos centramos en las restricciones en actividades de la vida diaria (AVD), ya que son las que generan mayores necesidades de cuidados personales, es decir mayor nivel de dependencia (de otras personas).

Las actividades de la vida diaria (AVD) tenidas en cuenta por la encuesta del Instituto Nacional de Estadística (INE) de 1999 son las siguientes: 1) realizar cambios y mante- nimiento de las diversas posiciones del cuerpo; 2) levantarse, acostarse, permanecer de pie o sentado; 3 ) desplazarse dentro del hogar; 4) deambular sin medio de transporte; 5) asearse solo, lavarse y cuidar el aspecto físico; 6) controlar las necesidades fisiológicas y utilizar solo el servicio; 7) vestirse, desvestirse y arreglarse; 8) comer y beber ; 9) encargarse de las compras y del control de los suministros y servicios; 10) encargarse de las comidas; 11) encargarse de la limpieza y el planchado de la ropa; 12) encargarse de la limpieza y mantenimiento de la casa y 13) encargarse del bienestar de los demás miembros de la familia.

Con el fin de poder comparar los resultados se han tomado las mismas actividades consideradas por el INE. Aunque las actividades relacionadas con las compras, los suministros y servicios, la elaboración de las comidas, la limpieza, el mantenimiento de la casa y el cuidado del bienestar de los demás miembros de la familia podrían clasificarse también como actividades instrumentales de la vida diaria (AIVD).

Sujetos de estudio: En España existen dos grandes tipologías de estudios muestrales extrapolables a población sobre la incidencia de la dependencia. Por un lado, estudios exhaustivos de diseño cuidado, con información comprehensiva pero de ámbito local como son las encuestas de $\mathrm{Vigo}^{16}$, Leganés y Móstoles ${ }^{17}$. Por otro lado, existen diferentes estudios de ámbito nacional, de los cuales la Encuesta de Discapacidades, Deficiencias y Estado de Salud (EDDES) proporciona la base estadística más actual y de mayor amplitud para todo el territorio español. La EDDES fue realizada por el INE durante el segundo trimestre de 1999 y en la misma se recoge información sobre personas residentes en viviendas familiares (es decir, se excluyen las personas institucionalizadas). Esta encuesta, con un amplio tamaño muestral (79.000 viviendas y 220.000 personas aproximadamente), abarcó todo el territorio español. 
En la encuesta se consideran 10 grupos de discapacidades y un total de 36 actividades. Según el INE, si la persona es capaz de realizar esas actividades sin ayudas técnicas externas ni ayudas personales, se considera no discapacitada. Es decir, una persona que requiera del uso de ayudas técnicas externas (como muletas, sillas de ruedas, etc.) es discapacitada. No obstante, si la discapacidad es superada con el uso de ayudas técnicas internas (marcapasos, lentes intraoculares, etc.) la persona no entra dentro de la categoría de discapacitado. En el caso de la visión se hace una excepción y sólo se recogen las discapacidades que subsisten con el uso de gafas y no aquéllas que hayan sido superadas con el uso de este tipo de ayudas técnicas, aunque sean externas.

La EDDES considera tres niveles de severidad: moderada, grave y absoluta, según el grado de dificultad que presente la persona para realizar la actividad. Además, en la encuesta se contempla una cuarta categoría, «sin dificultad», que corresponde a aquellas personas con discapacidades que han superado la restricción con el uso de alguna ayuda técnica (interna o externa). Para este trabajo, y por lo comentado anteriormente, dichas personas han sido consideradas como no dependientes. Así, para la valoración de los grados de dependencia a partir de dicha información tomamos los tres niveles de severidad mencionados de las trece AVDs descritas en el apartado anterior.

Cada persona puede presentar una discapacidad o más y en la EDDES se recogen todas las discapacidades que sufre una misma persona, así como el grado de dificultad en cada una de dichas actividades. Es por ello que para poder determinar el grado de dependencia de cada persona es necesario adoptar algún criterio que permita identificar el grado de severidad de cada individuo en función de las dificultades en las AVDs.

Definición de la tasa de prevalencia de la dependencia: El indicador utilizado en el análisis de la frecuencia de la dependencia es la tasa de prevalencia, estadístico habitualmente empleado en este tipo de estudios ${ }^{18,19}$. Los datos proporcionados por el INE permiten calcular las tasas de prevalencia para cada grado de severidad g, edad x y sexo s, a partir de la siguiente expresión.

$$
\lambda_{x, s}^{g}=\frac{\boldsymbol{N P D}_{x, s}^{g}}{\boldsymbol{P} \boldsymbol{T}_{x, s}}
$$

donde $N P D_{x, s}{ }^{g}$ es el número de personas dependientes de edad $\mathrm{x}$, sexo $\mathrm{s}$ y grado de severidad g. $P T_{x, s}$ es la población total de edad $\mathrm{x}$ y sexo $\mathrm{s}$.

Por tasa de prevalencia de personas dependientes de una determinada edad, sexo y grado de severidad entendemos la proporción de personas dependientes respecto al total de población de la misma edad y sexo.

Criterio de valoración de máxima severidad: El criterio de «máxima severidad» utilizado por el INE identifica el grado de severidad de la discapacidad de cada individuo, tomando en cuenta únicamente la gravedad de la AVD con mayor nivel de severidad. Consecuentemente, el INE asigna a cada persona un grado de discapacidad $G$, que se corresponde con el nivel de severidad correspondiente a la AVD de máxima gravedad, es decir:

$$
G=\operatorname{Max}\left\{V_{i}\right\}_{i=1, \ldots, 13},
$$

donde $V_{i}$, que representa el grado de severidad de la AVD i-ésima, puede tomar los siguientes valores: 0 si la persona es autónoma; 1 si la persona se considera discapacitada moderada; 2 si la persona sufre una discapacidad severa y 3 si la discapacidad es absoluta. Si se aplicase el mismo criterio para determinar el nivel de dependencia de una persona el mismo se correspondería con el grado de severidad de la discapacidad en las AVD con mayor índice de gravedad, obviando de esta forma la dificultad de las doce AVDs restantes. 
Criterio de valoración alternativo: Con el objeto de evaluar el nivel dependencia de las trece AVDs incluidas en la encuesta, se ha definido un índice de valoración global representado como I, que se obtiene sumando los grados de severidad de las trece AVD consideradas en la encuesta. El índice I, que, al igual que el índice de Barthel ${ }^{18}$ toma en cuenta todas las AVDs, ofrece la siguiente forma:

$$
I=\sum_{i=1}^{13} V_{i}
$$

Como resultado final, se ha elaborado un criterio de valoración de la dependencia basado en los índices $G$ e $I$, que tiene en cuenta el nivel de severidad de todas las AVDs consideradas en la encuesta. Su aplicación se articula a través de la siguiente formulación matemática: $\mathrm{Si} G=0$ la persona es autónoma; Si $G=1$ y $0<I<5$, la persona es dependiente moderada; $\mathrm{Si}(G=2$ y $0<I<11)$ o bien $(G=1$ y $5 \leq I \leq 13)$ la persona padece dependencia severa; $\mathrm{Si} \mathrm{G}=3$ o $(G=2$ y $11 \leq I$ $\leq 26$ ) la persona sufre dependencia absoluta.

Análisis de sensibilidad: Con el fin de analizar la influencia del cambio de criterio de valoración de la severidad de la dependencia sobre el comportamiento de las tasas de prevalencia de la dependencia resulta necesario realizar un ajuste por edades de los resultados obtenidos por ambos criterios. Una vez obtenidos los valores ajustados respectivos se compara el grado de significación de sus diferencias mediante intervalos de confianza. Para la estimación de los parámetros a partir de los valores logarítmicos de las tasas de prevalencia se ha utilizado el método de mínimos cuadrados ordinarios, especificando una función polinómica de grado $\mathrm{n}$ de la siguiente forma:

$$
\operatorname{Ln} \lambda^{g}{ }_{x, s}=\sum_{r=0}^{n} a_{r} \cdot x^{r}+\check{\boldsymbol{a}}_{x, S}^{g} \quad \operatorname{con} \dot{\boldsymbol{a}}_{x, s}^{g} \cong N\left(0, \sigma^{2}\right)
$$

donde los parámetros $a_{r}(r=0, \ldots . n)$ se estiman para cada grado de severidad y sexo. En cada uno de los procesos de ajuste, se han ensayado funciones polinómicas hasta el grado 6.

\section{RESULTADOS}

\section{Tasas de dependencia}

En las figuras 1 y 2 se representan las tasas de prevalencia por grado de severidad para hombres y mujeres de 50 años de edad en adelante, calculadas bajo el criterio de máxima severidad y bajo el criterio alternativo, respectivamente. En general, puede observarse que la tasa de dependencia sigue un crecimiento exponencial con la edad (bajo ambos criterios). Para las tasas de dependencia moderada y grave se observa un fuerte cambio de tendencia en los últimos tramos de edad. Este último comportamiento, especialmente marcado en la población femenina, obedece a que en edades avanzadas el grado de severidad de las discapacidades aumenta y, consecuentemente, se produce un trasvase de población desde el grupo de las personas con discapacidades moderadas y graves hacia el grupo de personas con discapacidades de máxima severidad. Como puede observarse en las figuras 1 y 2 , en los últimos tramos de edad existen fuertes oscilaciones de las tasas de dependencia, lo que en gran parte es debido a la falta de información para dichas edades. Con el objeto de efectuar el ajuste de las series, en primer lugar se calculan los logaritmos de las tasas, con el fin de suavizar las fluctuaciones y así atenuar sus efectos sobre las propiedades de los estimadores obtenidos en el ajuste.

\section{Ajustes}

En las tablas 1 a 4 se resumen los resultados obtenidos para los parámetros estimados, así como la significación individual (estadístico t-student) de cada uno de ellos. Puede observarse que los parámetros de las variables seleccionadas son claramente sig- 

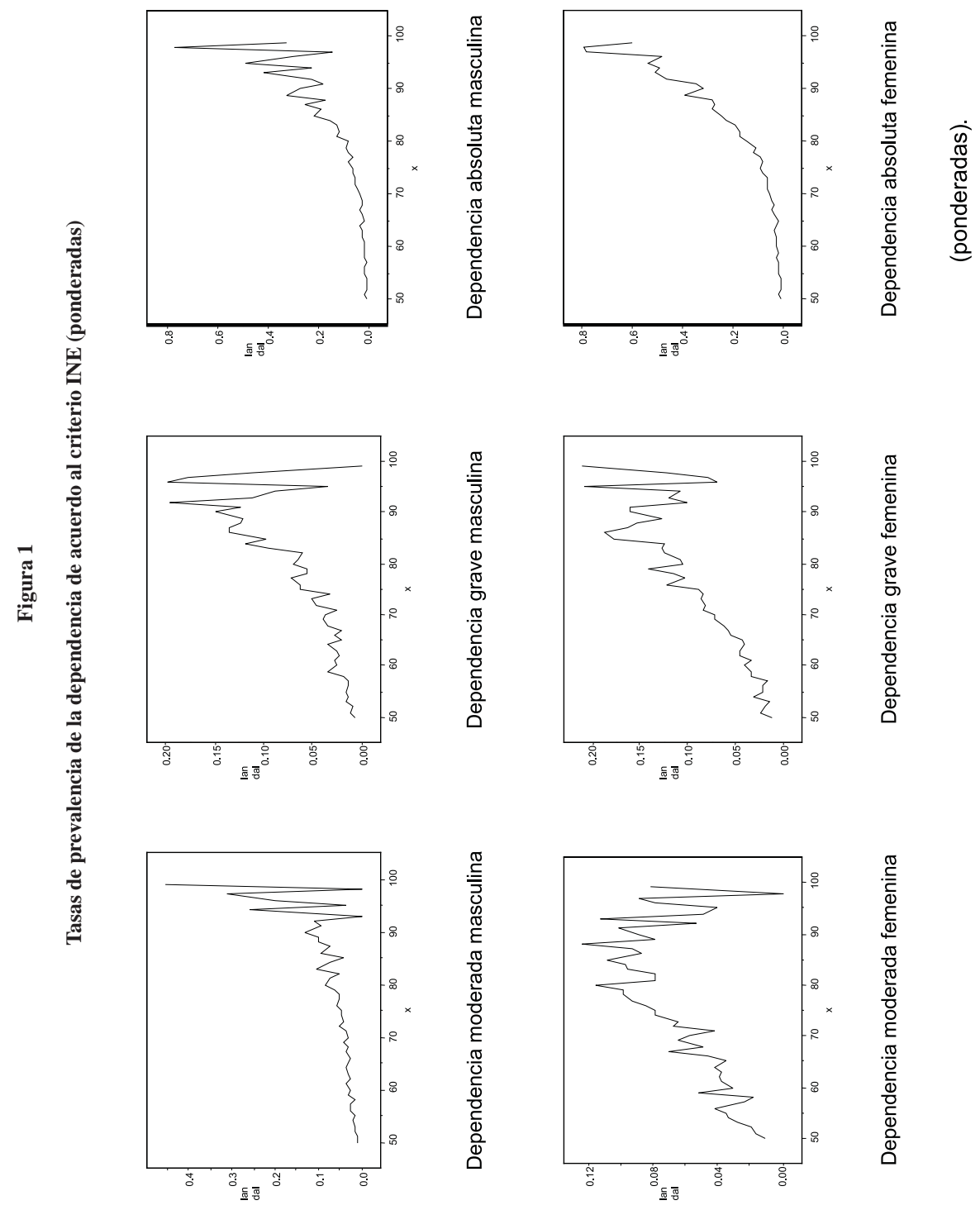

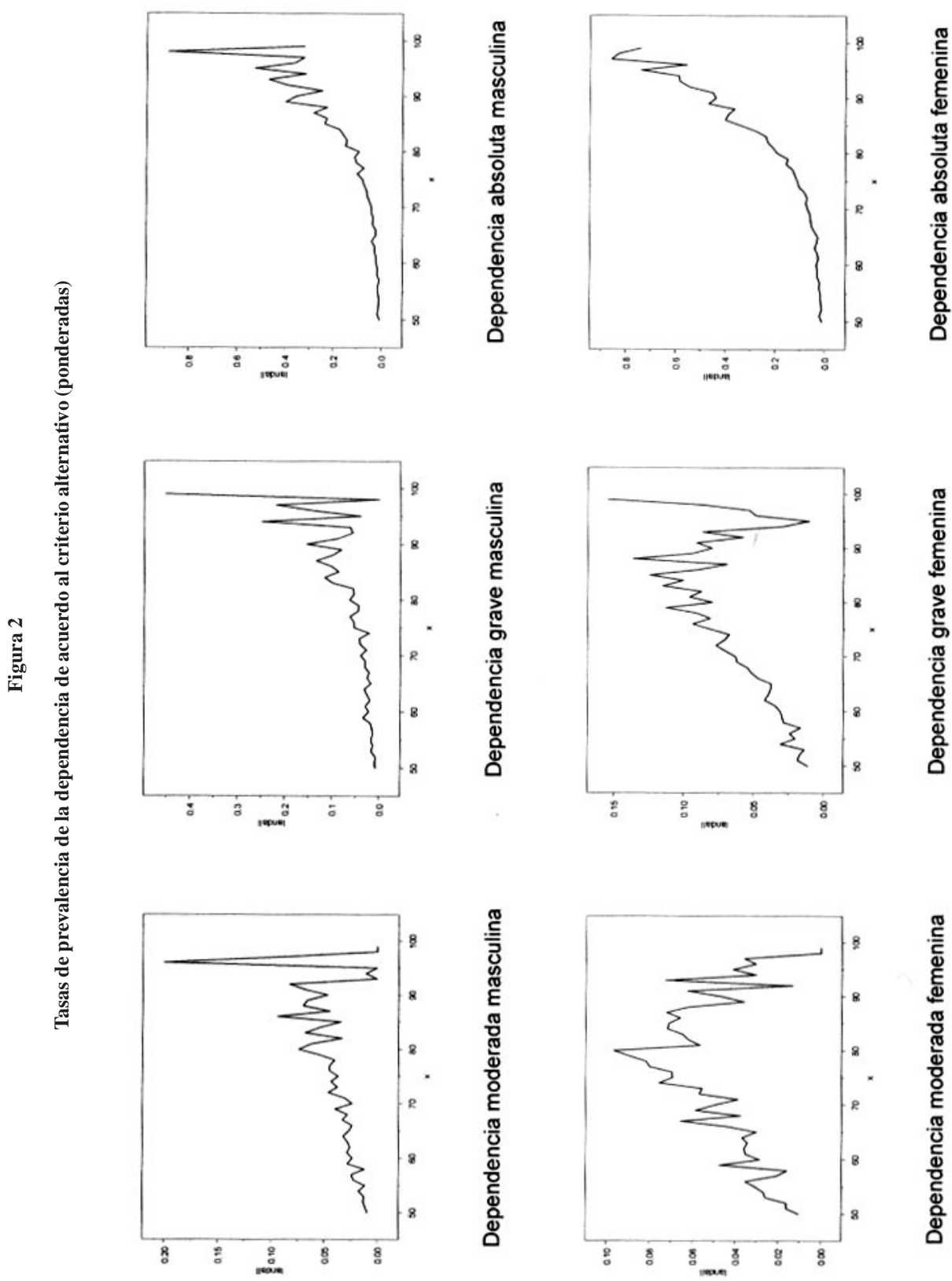
Tabla 1

Ajuste del In de las tasas de prevalencia masculinas. Criterio del INE

\begin{tabular}{|cccccc|}
\hline & $\mathrm{a}_{0}$ & $\mathrm{a}_{1}$ & $\mathrm{a}_{2}$ & $\mathrm{a}_{3}$ & $\mathrm{a}_{4}$ \\
\hline Dependencia & -30.711 & 1.085 & -0.015 & 0.000 & - \\
moderada & $(-5.607)$ & $(4.715)$ & $(-4.646)$ & $(4.810)$ & -0.000 \\
\hline Dependencia & -82.657 & 4.359 & -0.091 & 0.001 & $(-2.490)$ \\
grave & $(-2.544)$ & $(2.380)$ & $(-2.389)$ & $(2.438)$ & - \\
\hline Dependencia & -9.380 & 0.088 & & - & \\
absoluta & $(-50.435)$ & $(35.410)$ & & & \\
\hline
\end{tabular}

Ajuste por mínimos cuadrados ordinarios. Entre paréntesis se muestra el valor estimado del estadístico t-sudent.

Tabla 2

Ajuste del logaritmo neperiano de las tasas de prevalencia masculinas. Criterio alternativo

\begin{tabular}{|cccccc|}
\hline & $\mathrm{a}_{0}$ & $\mathrm{a}_{1}$ & $\mathrm{a}_{2}$ & $\mathrm{a}_{3}$ & $\mathrm{a}_{4}$ \\
\hline Dependencia moderada & -9.111 & 0.121 & -0.000 & - \\
& $(-7.477)$ & $(3.496)$ & $(-2.318)$ & \\
\hline Dependencia Grave & -7.541 & 0.059 & & - \\
& $(-29.264)$ & $(16.990)$ & & - \\
\hline Dependencia absoluta & -9.333 & 0.090 & - & - \\
\hline
\end{tabular}

Ajuste por mínimos cuadrados ordinarios. Entre paréntesis se muestra el valor estimado del estadístico t-sudent.

nificativos a nivel individual para un nivel de significación del 95\%. Además, cabe destacar que la bondad de ajuste de los modelos es igualmente satisfactoria. En los 12 ajustes realizados el coeficiente de determinación toma valores superiores a 0,75 . Por último, los diagramas de residuos indican la ausencia de heteroelasticidad y autocorrelación y, por tanto, cabe esperar que los estimadores cumplan con las propiedades deseables. 
Tabla 3

Ajuste del logaritmo neperiano de las tasas de prevalencia femeninas. Criterio del INE

\begin{tabular}{|c|c|c|c|c|c|}
\hline & $a_{0}$ & $a_{1}$ & $a_{2}$ & $a_{3}$ & $a_{4}$ \\
\hline \multirow[b]{2}{*}{ Dependencia moderada } & -11.883 & 0.220 & -0.001 & \multirow[b]{2}{*}{-} & \multirow[b]{2}{*}{-} \\
\hline & & & & & \\
\hline \multirow{2}{*}{ Dependencia grave } & -13.497 & 0.253 & -0.001 & \multirow[b]{2}{*}{-} & \multirow[b]{2}{*}{-} \\
\hline & $(-14.944)$ & $(10.059)$ & $(-8.175)$ & & \\
\hline \multirow[b]{2}{*}{ Dependencia absoluta } & -9.739 & 0.096 & \multirow[b]{2}{*}{ - } & \multirow[b]{2}{*}{-} & \multirow[b]{2}{*}{-} \\
\hline & $(-87.466)$ & $(66.575)$ & & & \\
\hline
\end{tabular}

Ajuste por mínimos cuadrados ordinarios. Entre paréntesis se muestra el valor estimado del estadístico t-sudent.

Tabla 4

Ajuste del logaritmo neperiano de las tasas de prevalencia femeninas. Criterio alternativo

\begin{tabular}{|cccccc|}
\hline & $\mathrm{a}_{0}$ & $\mathrm{a}_{1}$ & $\mathrm{a}_{2}$ & $\mathrm{a}_{3}$ & $\mathrm{a}_{4}$ \\
\hline Dependencia moderada & -14.421 & 0.296 & -0.002 & - \\
& $(-12.577)$ & $(9.236)$ & $(-8.583)$ & \\
\hline Dependencia grave & -14.825 & 0.296 & -0.002 & - \\
& $(-16.912)$ & $(12.114)$ & $(-10.630)$ & \\
\hline Dependencia absoluta & -10.446 & 0.127 & -0.000 & - \\
\hline
\end{tabular}

Ajuste por mínimos cuadrados ordinarios. Entre paréntesis se muestra el valor estimado del estadístico t-sudent.

\section{Análisis gráfico de la sensibilidad}

En la figura 3 se representan los ajustes de las tasas de dependencia para hombres y mujeres según grado de severidad, bajo el criterio de máxima severidad y bajo el criterio alternativo planteado. Además con líneas de puntos se representan los límites de los intervalos de confianza al $95 \%$ para el ajuste de la tasas de prevalencia bajo los dos criterios.

La figura 4 representa el cociente entre la tasa de prevalencia bajo el criterio alternativo y la tasa de dependencia bajo el criterio de máxima severidad. Valores alejados de 1 indican diferencias notables de las tasas entre ambos criterios. 

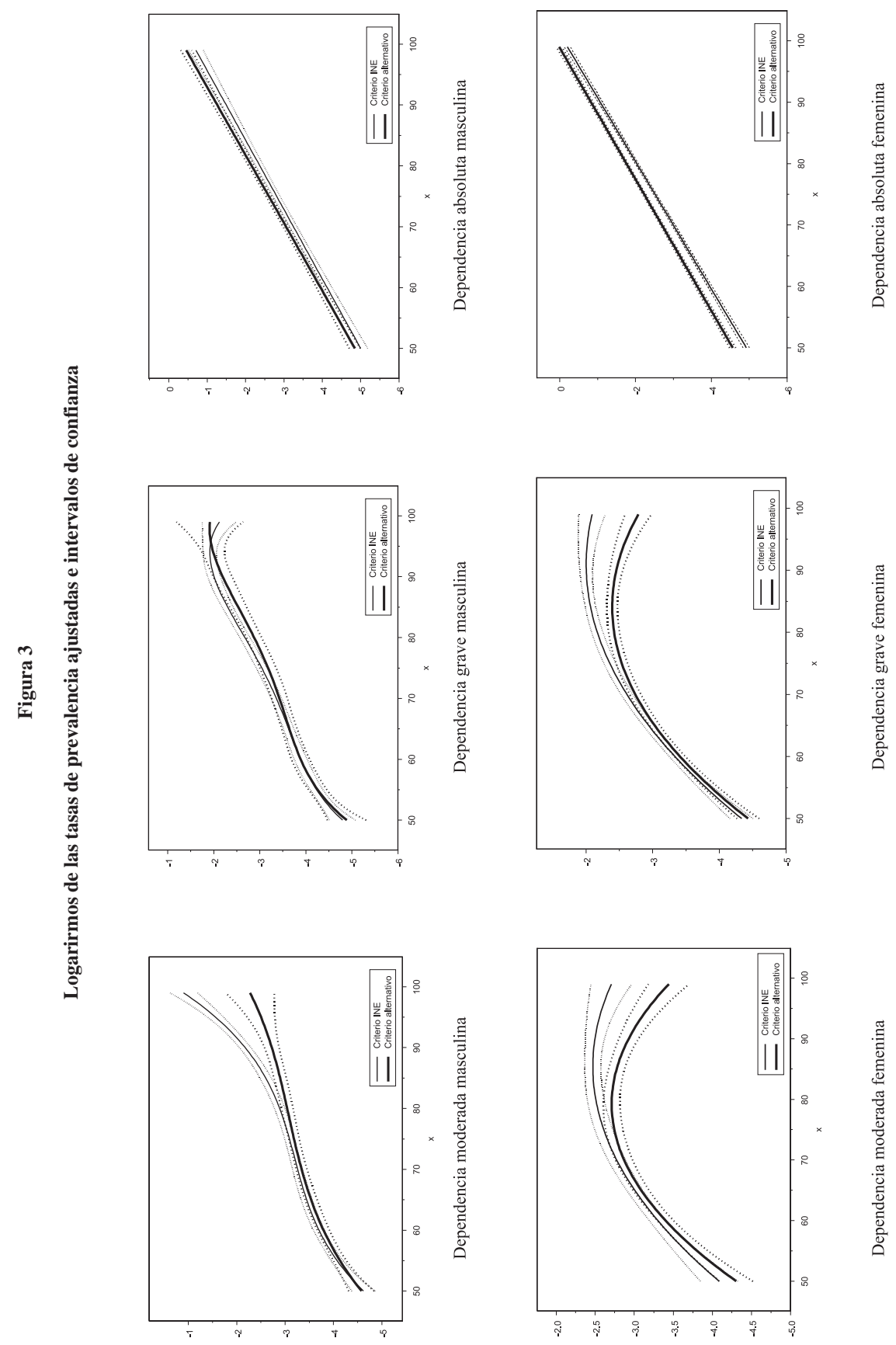
Figura 4

Razones entre tasas de prevalencia ajustadas por el criterio alternativo y criterio INE

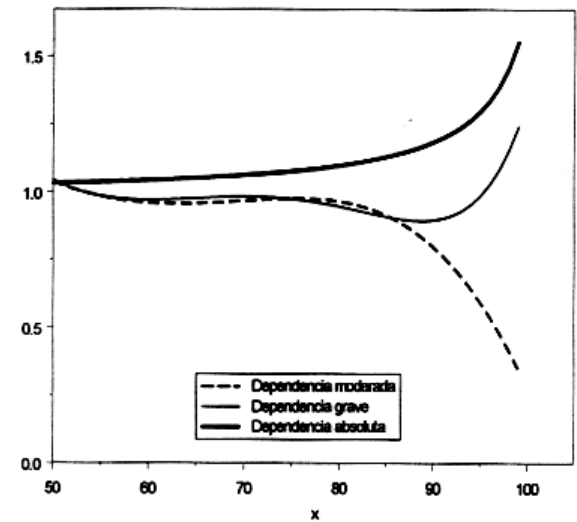

Población masculina

\section{DISCUSIÓN}

En todos los casos se observan diferencias significativas entre las tasas de dependencia dependiendo del criterio utilizado, en especial en los rangos de edades más avanzadas. Para los grados de dependencia menos severos (moderado y grave) las tasas calculadas en base al criterio de máxima severidad resultan más elevadas que bajo el criterio alternativo. Por el contrario, para las tasas de severidad absoluta se observa el comportamiento opuesto, es decir mayores tasas para el criterio alternativo.

Dicho comportamiento queda justificado por el hecho de que bajo el criterio alternativo las personas que presentan varias discapacidades menos severas (que bajo el criterio de la máxima severidad se englobarían en los menores grados de severidad) pueden pasar al grado superior de severidad como consecuencia de que se suman todas las discapacidades padecidas (AVDs). Por tanto, puede decirse que las tasas calculadas de acuerdo a la valoración de máxima severidad subesti-

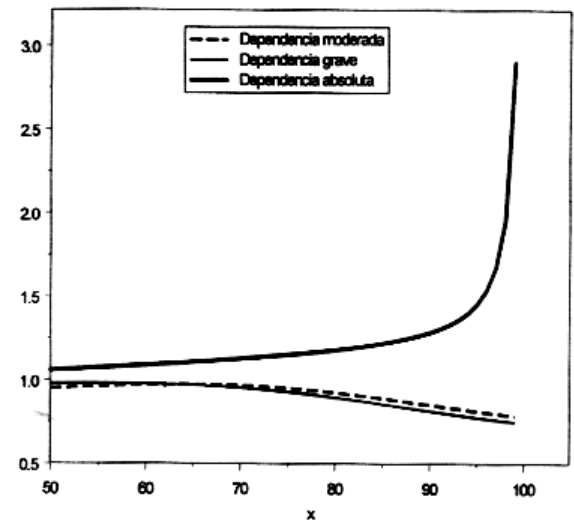

Población femenina

man la mayor dependencia ocasionada por un mayor número de discapacidades.

Por su parte, en cuanto al comportamiento funcional de las series, cabe destacar que a excepción de las tasas de dependencia masculina de grado moderado y grave, no se observan diferencias notables entre los criterios en cuanto a forma gráfica de los ajustes. En el caso de la población masculina el crecimiento de la tasa de dependencia moderada con la edad es menor bajo el criterio alternativo, debido al trasvase de población anteriormente explicado.

Del análisis del cociente entre la tasa de dependencia bajo el criterio alternativo y la tasa de dependencia del criterio de máxima severidad se observa que las diferencias crecen conforme aumenta la edad, como resultado de que el número de AVDs padecidas va aumentando, lo que es recogido únicamente por el criterio alternativo.

Por tanto, como conclusión cabe resaltar la existencia de diferencias notables de la 
prevalencia de la dependencia según sea el criterio seleccionado. Si bien dichas diferencias son menores en el tramo de edad de 50 a 70 años, a partir de esa edad el criterio de máxima severidad muestra una infravaloración sistemática en la tasa de dependencia absoluta, que es precisamente la que más afecta a la población de mayor edad. Una cifra que ayuda a comprender la magnitud de dicha subestimación es la cantidad de personas que quedan fuera de la categoría más grave de severidad bajo el criterio de máxima severidad respecto al criterio alternativo. Dicha cifra asciende a 141.755 personas, de las cuales 34.576 son hombres y 107.179 son mujeres.

Dado que la cuantificación de la dependencia constituye la base para la estimación de los costes asociados a los servicios de cuidados de larga duración ${ }^{20-22}$, merece ser destacada la importancia de introducir criterios de valoración objetivos ${ }^{22}$ basados en estudios gerontológicos y que logren captar con mayor exactitud el verdadero problema de la dependencia en las personas mayores.

En la actualidad existen varios criterios para la medición de la dependencia que se diferencian entre sí por el tipo de actividades consideradas, así como por la forma de agrupar las diferentes limitaciones y las categorías de severidad. Dos de los índices más ampliamente utilizados son el índice de $\mathrm{Katz}^{23}$ y el índice de Barthel ${ }^{14}$, ambos basados en las AVD. Sin embargo las actividades consideradas de la vida diaria no son exactamente las mismas. Las actividades consideradas en el presente trabajo (y clasificadas por el INE como AVD) son más amplias que en los dos casos anteriores, en especial respecto al índice de Katz (que considera sólo 6 AVD).

Como criterio alternativo no se consideró ninguno de los dos índices anteriores, no sólo por las diferencias en la definición de las AVD sino por las características propias de la información con la que se trabajó. Las principales diferencias son que en el índice de Katz sólo se toma en cuenta si la persona es dependiente o independiente para realizar cada una de las AVD. Por su parte, el índice de Barthel considera dos grados de severidad de la dependencia en cada una de las actividades, mientras que la EDDES nos permite distinguir entre tres niveles de severidad para cada unas de las AVD, siempre desde la propia percepción del individuo.

Más allá de los aspectos metodológicos que dificultan el cálculo de los dos índices anteriores a partir de los datos de la EDDES cabe resaltar aquí que el presente trabajo más que presentar un criterio definitivo de medición ha estado orientado a analizar la existencia de diferencias en la prevalencia de la dependencia según cuál sea el criterio utilizado.

Por último, hay que destacar que además de la severidad y el número de discapacidades (o codiscapacidades) un aspecto relevante en la medición más precisa del grado de dependencia de las personas es el peso diferencial que en la práctica tienen las restricciones para realizar las diferentes actividades de la vida diaria, el cual ya es tenido cuenta en Francia ${ }^{24}$ o en otros trabajos de nuestro país ${ }^{7}$. La cuantificación de tales pesos es una de las cuestiones que se espera abordar en futuros trabajos.

\section{BIBLIOGRAFÍA}

1. Casado D, López-Casanovas G. Vejez, dependencia y cuidados de larga duración. Situación actual y perspectivas de futuro. Colección Estudios Sociales Núm. 6, Fundación «la Caixa»; 2001.

2. Antares Consulting. Seguro de Dependencia. Caracterización del Mercado Español. Barcelona: UNESPA; 2001.

3. Moragas R. El reto de la dependencia al envejecer. Barcelona: Herder; 1999.

4. Instituto Nacional de Estadísticas. Proyecciones de población calculadas a partir del Censo 2001. [cita- 
do 1 de enero 2005]. Disponible en: http://www. ine.es/inebase

5. Alan Walker. Vejez y protección social a la dependencia en Europa. Madrid: Instituto de Migraciones y Servicios Sociales; 1999

6. Vicente A, Pociello E, Varea J. Cobertura de la dependencia: Una comparación internacional. Actuarios 2004; 22:1-23.

7. Viciana F, Hernández-Rodríguez JA, Conto VD y Ávila AL. Longevidad y calidad de vida en Andalucía. Sevilla: Instituto de estadística de Andalucía; 2003 [citado 1 de ene. 2005]. Disponible en: http://www.juntadeandalucia.es/institutodeestadistica/

8. Verbrugge LM, Jette AM. The disablement process. Soc Sci Med 1994; 38:1-14.

9. Otero Á, Zunzunegui MV, Rodríguez-Laso Á, Aguilar MD y Lázaro P. Volumen y tendencia de la dependencia asociada al envejecimiento en la población española. Rev Esp Salud Pública 2004; 78:201-13.

10. Séculi E, Fusté J, Brugulat P, Junca S, Rue M y Guillen M. Percepción del estado de salud en varones y mujeres en las últimas etapas de la vida. Gac Sanit 2001; 15: 217-23.

11. Rodríguez Cabrero G (coord.). La protección social de la dependencia. Madrid: Instituto de Migraciones y Servicios Sociales; 1999

12. Instituto Nacional de Estadísticas. Resultados de la Encuesta sobre Discapacidades, Deficiencias y Estado de Salud. Madrid; 2000 [citado 10 de feb. 2002]. Disponible en: http://www.ine.es/discapal discapamenu.htm

13. Pitacco E. Modelli attuariali per le assicuraioni sulla salute. Centro di Ricerche Assicurative Previdenziali dell'Università Bocconi. Milano: CERAP; 1995.
14. Mahoney FL and Barthel DW. Functional evaluation: The Barthel Index. Md State Med J 1965; 14: $5-61$.

15. Bone M. Trends in dependency among older people in England. London: HMSO; 1995.

16. Eiroa Patiño P, Vázquez-Vizoso FL, Veras Castro R. Discapacidades y necesidades de servicios en las personas mayores detectadas en la encuesta de salud OARS-Vigo. Med Clin(Barc) 1996;106:641-8.

17. Fundación Caja Madrid. Estudio sobre las necesidades de atención socio-sanitaria de las personas grandes del municipio de Móstoles. Madrid: Fundación Caja Madrid; 1994.

18. Bermejo F, Diaz J, Olazarán J, Rivera J, Trincado R y Fernández C. Prevalencia de demencia en una muestra poblacional de ancianos en medio urbano. Análisis preliminar. Neurología 1995; 10: 418.

19. Rickayzen BD and Walsh DEP. A multistate model of disability for the United Kingdom: Implications for future need for Long-Term Care for the Elderly. British Actuarial Journal 2002; 8, vol II: 1-47.

20. Blandeau J and Dubois D. Financing Old-age Dependency in Europe: Towards overall management of old age. The Geneva Papers on Risk and Insurance 1997; 22: 46-59.

21. Meredith J. A Markovian Analysis of a Geriatric Ward. Manage Sci 1973; $19: 604-12$.

22. Moragas R, Allué R. El coste de la dependencia al envejecer. Barcelona: Herder; 2003.

23. Katz S, Ford AB, Moscowitz RW, Jackson BA, Jaffe MW. Studies of illness in the aged. The index of ADL: A standardized measure of biological and psychosocial function. JAMA 1963; 185: 914-9.

24. Instituto de Migraciones y Servicios Sociales. La protección social a las personas mayores en Francia. Boletín sobre el envejecimiento. Perfiles y tendencias 2004; núm 10. 